\title{
Preface: Selected papers from MEDGEO 2019 conference on recent progress in medical geology
}

\author{
Ping Li $\cdot$ Robert Finkelman $\cdot$ Chaosheng Zhang
}

Accepted: 13 February 2021 / Published online: 26 February 2021

(C) The Author(s), under exclusive licence to Springer Nature B.V. part of Springer Nature 2021

The 8th International Conference on Medical Geology was successfully held during August 12-15, 2019, in Guiyang, China. The Institute of Geochemistry, Chinese Academy of Sciences (IGCAS) and the International Medical Geology Association (IMGA) hosted the conference with the support of Guangzhou University, China University of Geosciences (Wuhan), National University of Ireland, Galway, and Guizhou Medical University. The IGCAS was founded in 1966, and now is one of the world leading research institutions in environmental geochemistry, ore deposit geochemistry, experimental geochemistry, and lunar and planetary sciences. The IMGA aims to

\section{P. Li $(\square)$}

State Key Laboratory of Environmental Geochemistry Institute of Geochemistry, Chinese Academy of Sciences, Guiyang, People's Republic of China

e-mail: liping@mail.gyig.ac.cn

\section{R. Finkelman}

Department of Geosciences, University of Texas at Dallas, Richardson, TX, USA

R. Finkelman

College of Geoscience and Survey Engineering, China University of Mining and Technology (Beijing), Beijing, China

\section{Zhang}

International Network for Environment and Health, School of Geography and Archaeology \& Ryan Institute, National University of Ireland, Galway, Ireland provide a network and a forum for scientists interested in characterizing geological processes, and the dispersal of geological material that effect the health of human populations.

About 300 experts and scholars from 23 countries, 80 universities and research institutes attended the conference. The theme of the conference was "medical geology and ecological civilization construction." The conference set up 7 Special Symposia, including Environmental geochemistry and human health, Water and human health, Soil pollution and remediation, Atmosphere pollution and human health, Lead in the Environment and Public Health, Mercury contamination and Human Health, and Numerical and Statistical Modeling and its Applications in Medical Geology The conference greatly promoted the cross integration of related disciplines and medical geology. The conference offered 6 plenary lectures, 80 oral presentations, and 115 poster presentations. The success of the conference played an important role in the development of medical geology and promoted international cooperation, marking a milestone in the development of medical geology in China and elsewhere.

The theme of this special issue is "Recent Progress in Medical Geology." This special issue collected 14 research papers and 1 review paper, which covered the topics of water pollution, soil pollution, and human exposure and health effects. 
1. Water pollution. Yuan et al. assessed heavy metals contamination and water quality characterization in a city river. Zhang et al. evaluated dissolved organic matter (DOM) sources in groundwater through the use of carbon isotopes, which is significant for predicting As fluctuation in aquifers affected by monsoon climate and/or human activities. Xiao et al. revealed the impact of the aquitard to As accumulation of underlying aquifer from central Yangtze River Basin. Sun et al. integrated the mass balance models of multiple tracers to understand the role of groundwater in maintaining the water balance and pollution status of Dongting Lake. Kousa et al. evaluated the variation of $\mathrm{Mn}$ concentrations in groundwater and factors in Finland.

2. Soil pollution. Qiu et al. found that anthropogenic $\mathrm{Pb}$ mainly exists in the soils of the lower riparian zone in the Three Gorges Reservoir, and the frequent and anti-seasonal dry and rewetting alternation aggravating the potential for $\mathrm{Pb}$ migration downstream. Zhong et al. revealed the distribution, origin, and speciation of soil Se in the black soil region of Northeast China. He et al. evaluated fluorine pollution in vegetables and soil around an abandoned aluminum plant. Han et al. evaluated soil polybrominated diphenyl ethers pollution and temporal trends in Tianjin City, North China. Bai et al. investigated the effects of
Cd pollution on $\mathrm{N}$ mineralization in urban constructed wetland soils of the Pearl River Delta by an aerobic incubation experiment. Tong et al. confirmed microbial community changes during microbial microaerophilic Fe(II) oxidation in paddy soil. Wang et al. provided new perspectives on metabolism pathways of salt tolerance in two wetland plants.

3. Human exposure and health effects. Chen et al. assessed geochemical characters of typical hot springs in Guizhou and analyzed the correlation between hot spring types and health. $\mathrm{Xu}$ et al. assessed the potential value of Rosa Roxburghii Tratt in arsenic-induced liver damage based on elemental imbalance and oxidative damage. $\mathrm{Wu}$ et al. reviewed the role of $\mathrm{p} 38$ signaling pathway in arsenic-induced apoptosis.

By presenting the links between environmental pollution and geological processes on human health, this special issue contributes to progress in medical geology. All the papers in this special issue were processed with regular peer review. We thank all the authors and reviewers for their efforts involved in this special issue.

Publisher's Note Springer Nature remains neutral with regard to jurisdictional claims in published maps and institutional affiliations. 